\title{
Behavioral evidence for task-dependent "what" versus "where" processing within and across modalities
}

\author{
Jason S. Chan ANd Fiona N. Newell \\ Trinity College Dublin, Dublin, Ireland
}

\begin{abstract}
Task-dependent information processing for the purpose of recognition or spatial perception is considered a principle common to all the main sensory modalities. Using a dual-task interference paradigm, we investigated the behavioral effects of independent information processing for shape identification and localization of object features within and across vision and touch. In Experiment 1, we established that color and texture processing (i.e., a "what" task) interfered with both visual and haptic shape-matching tasks and that mirror image and rotation matching (i.e., a "where" task) interfered with a feature-location-matching task in both modalities. In contrast, interference was reduced when a "where" interference task was embedded in a "what" primary task and vice versa. In Experiment 2, we replicated this finding within each modality, using the same interference and primary tasks throughout. In Experiment 3, the interference tasks were always conducted in a modality other than the primary task modality. Here, we found that resources for identification and spatial localization are independent of modality. Our findings further suggest that multisensory resources for shape recognition also involve resources for spatial localization. These results extend recent neuropsychological and neuroimaging findings and have important implications for our understanding of high-level information processing across the human sensory systems.
\end{abstract}

It is a commonly held view that perceptual systems share organizational principles of information processing specifically, that different resources deal with information for the purpose of recognition and spatial perception or action (Poremba et al., 2003; Reed, Klatzky, \& Halgren, 2005; Romanski et al., 1999; Ungerleider \& Mishkin, 1982). Moreover, it is presumed that this common division of labor facilitates the sharing of information across modalities, resulting in a coherent perception of the world. Very little evidence has been provided, however, to suggest that this type of organization of function has a direct effect on perceptual performance in neurologically intact individuals. Here, we investigated whether or not this independent processing of object and spatial information has an effect on visual and haptic perception and, if so, whether these effects are observable across modalities.

Converging evidence from neurophysiological, neuropsychological, and neuroimaging literatures has supported the idea of a separation of function, particularly within the primate visual system. Object recognition (the so-called "what") and spatial (the so-called "where" or "how") pathways were first mapped in the primate brain along the ventral and dorsal visual streams, respectively (Desimone, Schein, Moran, \& Ungerleider, 1985; Desimone \& Ungerleider, 1986; Felleman \& Van Essen, 1991; Mishkin, Ungerleider, \& Macko, 1983; Ungerleider, Galkin, \&
Mishkin, 1983). For example, Ungerleider and Mishkin reported that ablation of the inferotemporal cortex (ventral stream) of the macaque brain resulted in selective impairment of shape identification, whereas ablation of the posterior parietal region (dorsal stream) resulted in selective impairment of visuospatial perception. Evidence for a separation of function in the human visual system along homologous dorsal and ventral streams has mainly come from neuropsychological (e.g., Goodale \& Milner, 1992; Milner \& Goodale, 1995; Newcombe \& Russell, 1969) and neuroimaging (Haxby et al., 1991; Haxby et al., 1994; James, Culham, Humphrey, Milner, \& Goodale, 2003) studies. Interestingly, however, evidence is emerging to suggest that both the human auditory (Belin \& Zatorre, 2000; Clarke et al., 2002) and the somatosensory (Reed \& Caselli, 1994; Reed, Caselli, \& Farah, 1996; Reed et al., 2005) systems are similarly organized. Taken together, these findings strongly suggest that a common functional architecture underlies the organization of information processing in the brain.

If, as both the neuroimaging and the neuropsychological evidence suggests, the processing of information for recognition and spatial localization occurs independently, associated behavioral effects should be observable. Moreover, if such task-dependent processing of information occurs in the haptic system, the behavioral effects should 
generalize from vision to touch. To date, however, very little behavioral evidence exists for how performance is related to the division of labor within the visual and tactile perceptual systems. It also remains an empirical question whether such functional organization facilitates perception across these modalities.

There are reasons to suggest that task-dependent effects on behavior may not be found. For example, the findings of some studies suggest that the cortical ventral and dorsal streams of the visual system are not completely functionally independent but that different tasks can involve activation in different areas associated within a common network (e.g., Marois, Leung, \& Gore, 2000; Merigan \& Maunsell, 1993). A similar finding was found for identification and spatial localization tasks in the tactile system: Although different cortical areas were activated by each task, these areas were not strictly defined along ventral and dorsal routes (Reed et al., 2005). On the other hand, neuropsychological evidence strongly suggests that cortical damage can result in selective impairment to performance in one of these tasks while performance in the other remains intact (Goodale \& Milner, 1992; Landau, Hoffman, \& Kurz, 2006; Reed \& Caselli, 1994; Reed et al., 1996).

Some studies have reported differential effects on behavioral performance between identification and spatial (i.e., "what" and "where") tasks within the visual domain (Irwin \& Brockmole, 2004; Logie \& Marchetti, 1991; Tresch, Sinnamon, \& Seamon, 1993). Tresch et al., for example, employed a dual-task interference paradigm, where participants were visually presented with an object identification or spatial-matching interference task embedded in either an identification or a spatial primary task. They found that if the primary and interference tasks recruited the same resources (e.g., both "where"-based spatial tasks or both "what"-based identification tasks), the interference task significantly reduced accuracy on the primary task. On the other hand, if the primary and interference tasks were different (e.g., a "what"-based primary task and a "where"-based interference task), the interference task had little effect on primary task performance. Tresch et al. argued that since shape and spatial processing in the visual system do not interfere with each other, their results provide evidence for task-specific "what" and "where" resources.

Many recent behavioral studies have suggested that information encoded by one modality can be efficiently shared by another modality. For example, it is known that the recognition of objects can be primed across vision and touch (Easton, Srinivas, \& Greene, 1997; Reales \& Ballesteros, 1999) and that explicit cross-modal object recognition is efficient (Newell, Ernst, Tjan, \& Bülthoff, 2001). On the whole, therefore, these findings suggest that at least some resources for processing "what" information, or object identification, are shared across the visual and haptic modalities.

Evidence for shared processing of spatial information across modalities is, however, less forthcoming. A recent neurophysiological study suggests that, in the monkey at least, sensory-specific spatial information is encoded into different reference frames, so that spatial information is not necessarily integrated (Avillac, Denève, Olivier, Pouget, \& Duhamel, 2005). Avillac et al. reported that cells within the ventral intraparietal area are selectively tuned to spatial information encoded by either vision or touch. The few behavioral studies of cross-modal spatial perception that have been reported have also suggested that spatial information is specific to each sensory modality (e.g., Kappers, 1999; Newell, Woods, Mernagh, \& Bülthoff, 2005). For example, Newell et al. (2005) reported that the recognition of the spatial layout of a set of familiar objects is better within sensory modalities than across modalities, suggesting that representations of spatial information are sensory specific. However, differences between the modalities may be specific to largescale spatial layouts, where vision may dominate, and may not be observed in tasks involving small-scale (i.e., hand-sized) spatial events, where both modalities may be equally efficient.

Other studies suggest that spatial information is indeed shared across modalities (Kerzel, 2001; Smyth \& Pendleton, 1989). Kerzel, for example, found that the rate at which a person moved his or her hand could interfere with the memory for the rate of a moving visual stimulus. Although these studies do not provide direct evidence for whether spatial information is shared across vision and touch, since the tasks largely involve kinesthetic, rather than tactile, processing, they suggest that this sharing is nevertheless possible.

In the following experiments, we tested for behavioral evidence for a separation of function between "what" and "where" tasks within and across vision and touch. In our experiments, we adapted the methodology used by Tresch et al. (1993) in their study of separation of function within the visual domain. Specifically, we used a dual-task interference paradigm to investigate the behavioral effects of independent processing of information for shape recognition and spatial location. In the last experiment reported here, we investigated "what" versus "where" processing across modalities. To that end, we further modified the Tresch et al. paradigm so that the same tasks and stimuli were used throughout the experimental conditions. Tresch et al. used different stimuli and tasks across the primary and interference tasks (i.e., shape and color discrimination vs. localization and motion perception); therefore, it is not known to what extent the results were affected by top-down changes in task set or attentional load, rather than by processes involved in object and spatial memory per se. These effects may be further compounded in a cross-modal paradigm. To avoid this concern, in the cross-modal experiment, we used the same stimuli interchangeably across conditions, and the task was based on a same/different paradigm irrespective of condition. An overall matching paradigm was deliberately used across both the primary and the interference tasks. By presenting a task with the same response criteria, our aim was to minimize the possibility of introducing higher cognitive interference due to task switching (Kinsbourne, 1980) or demands on attentional resources (see Hirst \& Kalmar, 1987). The goal of the interference task was thus to determine whether the perceptual resources involved in shape 
recognition are also involved in local feature location and vice versa, without recourse to changes in the cognitive demands of the task.

To verify our paradigm, however, we initially tested the effect of different stimuli in the interference task (e.g., color and rotation matching for the identification and spatial tasks, respectively, in the visual condition and texture and orientation matching in the tactile condition) on primary task performance.

\section{GENERAL METHOD}

\section{Stimuli and Apparatus}

See Figure 1 for an illustration of the stimuli used and an example of the trial structure in the experiments. Ten unique and unfamiliar configurations of Lego bricks were designed as stimuli, with the constraint that each object was made of three to four parts and the entire object was hand-sized. The maximum dimensions of the objects were $8 \times 8 \mathrm{~cm}$. A flat (i.e., smooth) $1 \times 0.5 \mathrm{~cm}$ Lego tile was randomly placed upon the surface of each of the Lego shapes. We created copies of these shapes, yielding a total of eight shape copies per object. A Lego tile was then placed on the surface of each object. Of the eight copies of each shape, four were physically identical both in shape and in the position of the surface tile. For the other four shape copies, the tile was randomly located on the surface of the objects.

Each object was then fixed onto a wooden plate measuring $20 \mathrm{~cm}$ in diameter, which, in turn, lay flat on a table during the experiment. In order to randomize the position of the object on the circular plate, we used the four quadrants of far left, far right, near left, and near right with respect to the participant as position guides. Thus, within an object set, each stimulus copy was pseudorandomly positioned on a new wooden plate across the four quadrants, ensuring that, for all object pairs in a trial, the objects were always in a different position on the wooden plate. We did this in order to minimize any apparent motion cues in the visual stimuli that could signal a change in shape or location from one image to the next. Changes in orientation of the object stimuli within a trial did not occur.

Digital images of all 80 objects were used as stimuli in the visual conditions (i.e., in Experiments 1, 2A, and 3). The overall size of the images (i.e., plywood circle and Lego object) presented on the computer screen was $20 \mathrm{~cm}$ in diameter. These digital pictures were imported to Photoshop (Adobe Systems, San Jose, CA) and were converted to grayscale images. The luminance contrast between the plywood circle, Lego objects, and Lego tile was manipulated (plywood circle, FL24.97; Lego object, FL19.47; Lego plate, FL17.73).

The apparatus consisted of a 21-in. CRT computer monitor, mounted on top of an object occluder, and the same apparatus was used in all the experiments. In Experiments 1, 2A, and 3, the visual

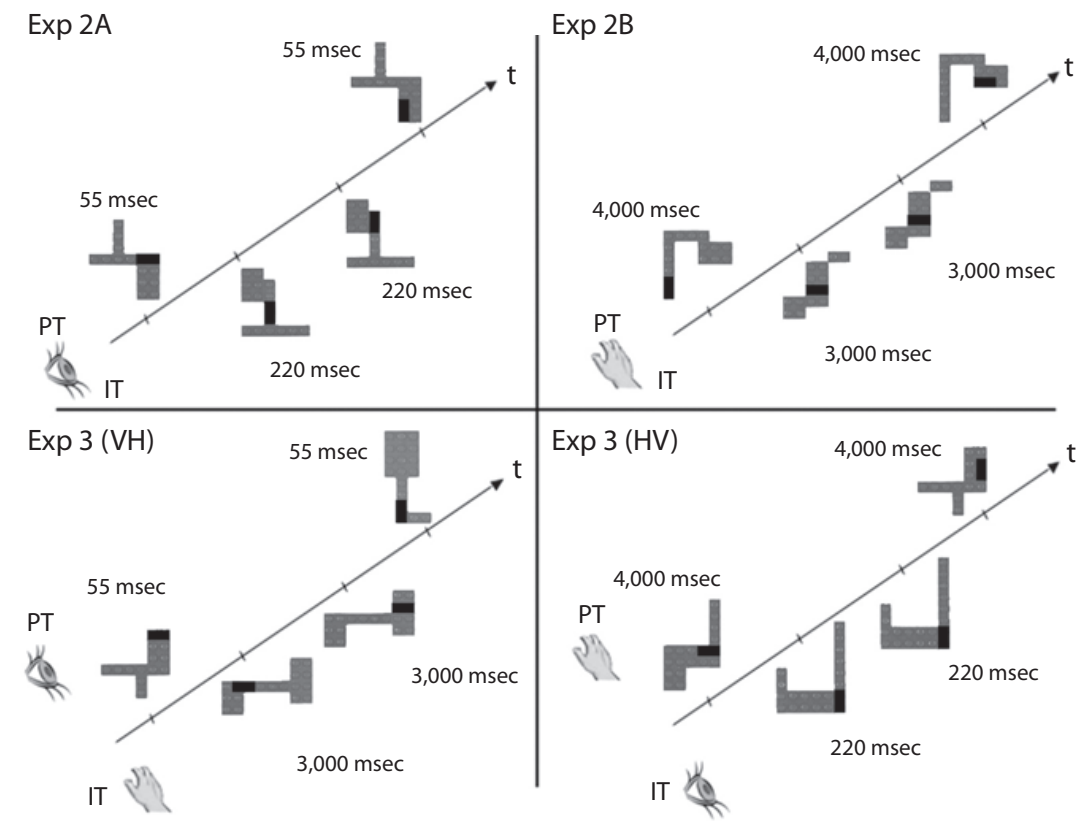

Figure 1. Schematic example of the temporal sequence of stimulus events during a typical trial consisting of a shape primary task and a location interference task in each of our Experiments 2A, 2B, and 3. Within each panel, the stimuli are presented along a time line in right-to-left order from the first stimulus presented in the primary task (PT; i.e., S1) to the last stimulus in the PT (i.e., S4), with the stimuli in the interference task (IT) in between (i.e., S2 and S3). For illustrative purposes, the stimuli positioned above the time line represent those involved in the PT (i.e., S1 and S4), and stimuli positioned below the time line represent those in the IT (i.e., S2 and S3). The presentation times are presented beside each stimulus. (For ISI information in each experiment, please see the main text.) The top two panels depict same-shape PT trials from our unimodal experiments (2A and $2 B$ ), and the bottom two panels represent different-shape trials from the cross-modal conditions in Experiment 3 (visual-haptic $[\mathrm{VH}]$ and Haptic-Visual $[\mathrm{HV}]$, respectively). Similarly, in the left-hand panels, the location IT stimuli are different, whereas in the right two panels, the location IT stimuli are the same. The stimuli in this depiction were randomly chosen from our full set for illustrative purposes only. 
stimuli were presented on the computer monitor that was placed directly in front of the participant at a distance of approximately $57 \mathrm{~cm}$. Thus, each visual stimulus subtended approximately $10^{\circ}$ of visual angle (the Lego object subtended approximately $4^{\circ}$ of visual angle). In Experiments 1, 2B, and 3, the haptic stimuli were presented on the table directly in front of them, behind the occluder. The occluder surrounded the haptic stimulus, so that the participants were unable to see the object. The participants were instructed to place their preferred hand behind the occluder to feel the stimulus.

Voice-activated responses were recorded using a head-mounted microphone. A custom-built speech recognition program was integrated into the experimental protocol, which was designed to recognize the words same and different only from each participant. The voice-activated recording program was calibrated to each participant's voice before the experiment began. The participants' responses were automatically recoded to text for analysis. If the program could not understand a participant's response, it would ask him or her to state the response again. This occurred on fewer than $1 \%$ of the trials within any one experiment.

\section{Procedure}

We used both a recognition and a localization task to tap the socalled "what" and "where" resources, respectively. Importantly, the task in all the experiments was a matching task, irrespective of modality. All the experiments reported here were based on a dual-task interference paradigm in which participants had to perform an interference task embedded in a primary task. Thus, the two different tasks (primary and interference) took place within each trial.

The recognition primary task involved global shape matching, and the localization primary task involved matching the location of a local feature (i.e., a smooth Lego tile) across objects. For simplicity, a task involving processing for recognition was labeled as a shape task, and a task involving spatial processing was labeled as a location task, here and in all the subsequent experiments. In Experiment 1 , our interference tasks involved tasks that were different from the primary tasks but were again labeled "what" or shape tasks (involving color or texture matching) and "where" or location tasks (involving mirror image and orientation matching). In the location trials, the object shape remained the same throughout the block; therefore, shape information was redundant in the location task. Similarly, although all the stimuli contained a Lego tile, its position was noninformative during the shape tasks. ${ }^{1}$

For the location task, it was necessary to use local feature matching to avoid gross changes in eye or hand movements across stimuli that might effect changes in the nature of the information encoded (e.g., changes in scale, spatiotemporal encoding, or kinesthetic information). Localizing a feature within the frame of an object is an often-used spatial task that is known to activate cortical areas not involved in object recognition per se (Duncan, 1984; Egly, Driver, \& Rafal, 1994; Humphreys \& Riddoch, 2003; Liu, Slotnick, Serences, \& Yantis, 2003; Serences, Schwarzbach, Courtney, Golay, \& Yantis, 2004; Vecera \& Farah, 1994). Furthermore, object-based feature location tasks are ubiquitous in everyday perceptual tasks: To interact appropriately with an object - a mug of coffee, for example - it is first necessary to locate the handle of a mug in order to lift it. Note that recognition of an object is not necessary for the perception of spatial information for action (Goodale \& Milner, 1992).

Within a trial, the participants were presented with the first stimulus (S1) of the primary task (shape or location) and were instructed to remember the stimulus because they would have to compare it with another stimulus later on. The participants were then required to perform the interference task (i.e., matching S2 and S3). Shape and location tasks were identical in design, irrespective of whether they were primary or interference tasks. During the interstimulus interval (ISI) between S3 and S4, the participants were required to respond to $\mathrm{S} 3$, and feedback was given immediately after this response. Feedback was given with respect to their performance on the interference task to ensure that the participants were performing the task and that the same amount of cognitive effort was main- tained across the interference tasks throughout the experiment. In all the experiments, the ISI between $\mathrm{S} 3$ and $\mathrm{S} 4$ was at least $1 \mathrm{sec}$ in visual trials and $5 \mathrm{sec}$ in haptic trials, although a delay in responding increased this ISI. On average, responses and feedback occurred within this ISI. After the interference task, the participants were presented with the second stimulus (S4) of the primary task, to which they had to respond. Therefore, the participants were required to recall the first stimulus (S1) presented in the primary task condition and compare it with the last stimulus in the trial (S4) with respect to shape or location.

During the haptic blocks in the experiments, a 50-msec auditory "click" alerted the participants to begin palpating the object. They felt the stimulus with their preferred hand until the auditory click was once again presented. At that time, the participants removed their hand from the object, and the experimenter immediately removed the haptic stimulus and replaced it with the next haptic stimulus. There was a $25-\mathrm{sec}$ intertrial interval (ITI). This delay was required so that the experimenter would have sufficient time to prepare the stimuli for the next trial. There was also a 5,000-msec ISI to give the experimenter time to replace the previously felt stimulus with the new stimulus within a trial. This time also made it possible for the participant to respond to the interference task (i.e., matching S2 and S3) and for feedback to occur to that response before S4 was presented.

Trials were blocked according to the nature of the primary and interference tasks. Thus, there were always at least four blocks of trials in each experiment (i.e., four combinations of primary and interference shape and location tasks). The participants received a practice block of four random trials prior to testing in each block. The experiment proceeded once the participant and the experimenter were satisfied that the instructions and the protocol were fully understood. Otherwise, the practice trials were repeated. The participants were informed of the nature of the primary and interference tasks prior to each block. Feedback was not given on their responses to the primary task, although the participants were instructed to perform as quickly and as accurately as possible to both the primary and the interference tasks. Accuracy rates were recorded for all the tasks.

\section{EXPERIMENT 1}

In this experiment, we investigated whether resources for object recognition and spatial localization are independent in both the visual and the touch modalities. Previous behavioral evidence has suggested independence of resources within the visual domain (e.g., Tresch et al., 1993), although evidence for similar information processing in the tactile system has been less forthcoming. Our aim was to find evidence for a dissociation between shape and location processing at the perceptual level in both the visual and the tactile systems. We reasoned that if associated resources are independent, processing in one system should not interfere with processing in the other.

The "what" or shape interference tasks in this experiment were color matching and texture matching across vision and touch, respectively. For the "where" or location interference tasks, we used mirror image and rotation matching in the visual condition and stimulus orientation matching in the tactile condition.

\section{Method}

Participants. Twenty-four participants took part in this experiment (15 of them female and 9 male with an age range of 19-31 years and a mean age of 23.22 years). All the participants reported normal or corrected-to-normal vision and no tactile impairments. Three participants reported being left-hand dominant, and the rest reported being right-handed. The task (and all the subsequent ex- 
periments reported here) was approved by the School of Psychology Ethics Committee at Trinity College. In accordance with this approval, written consent was provided by each participant before the experiment began.

Stimuli. The stimuli in the primary task were the same as those described in the General Method section. We created two different sets of stimuli for the visual and tactile interference tasks. The visual interference stimuli consisted of a set of $3 \times 3$ matrices made up of colored patches. Pairs of color stimuli were created by making slight differences in hue to the central patch (i.e., by making a $5 \%$ color change in the red, magenta, cyan, or yellow color level, using Photoshop) or by mirror reversing and rotating the arrangement of the surrounding patches. The tactile interference stimuli consisted of a set of $3 \times 0.5 \mathrm{~cm}$ strips of sandpaper of various grades $(60,80$, and 100 grit) and positioned in different orientations $\left(70^{\circ}, 80^{\circ}\right.$, or $\left.90^{\circ}\right)$ on a cardboard background.

Design. The design of this experiment was based on a three-way repeated measures design with modality (vision or touch), primary task (shape or location), and interference task (shape or location) as factors. The resulting eight different experimental blocks were grouped into four blocks involving the visual modality and four involving touch. All the blocks were presented in an order that was counterbalanced across participants.

Procedure. The procedure generally followed that outlined in the General Method section, with the following exceptions. Here, the visual shape interference task was a color-matching task, and the participants were required to match the color of the central patch in the $3 \times 3$ array across sequential images. For the visual location interference task, the participants were instructed to ignore the central color patch and to match the arrangement of the surrounding color patches. The task was to decide whether these color patches were simply mirror reversed or were mirror reversed and rotated. In the tactile condition, the participants were required to match the roughness of the tactile stimuli in the shape interference task. For the location interference task, the participants were required to ignore texture differences and match the orientation of the tactile stimuli.

The timings for the shape primary task were 100 and 4,000 msec for the visual and tactile stimuli, respectively. Timings for the location primary task were 220 and 3,000 msec for vision and touch, respectively. Each visual stimulus in the shape interference task was presented for $75 \mathrm{msec}$, whereas those in the location interference task were presented for $140 \mathrm{msec}$. The stimuli in the tactile shape interference task were presented for $3,500 \mathrm{msec}$, and those in the location interference task were presented for 3,000 msec. These timings for the stimuli in the visual and haptic conditions were determined through pilot testing to ensure that performance would be similar between tasks and across modalities. The experiments took approximately $75 \mathrm{~min}$ to complete.

\section{Results}

We wanted to ensure that the participants were performing the interference task during the experiment, and not just the primary task. To ensure that this was the case, we set a $60 \%$ accuracy response criterion for the interference task in each modality and each block. The participants who did not achieve this level of performance were excluded from analysis. Consequently, 8 of the participants failed to reach this level of performance; therefore, their data were excluded from further analyses.

We conducted a three-way repeated measures ANOVA on the accuracy performance for the primary task, with modality (visual or haptic), primary task (shape or location), and interference task (shape or location) as factors. There was no main effect of modality $[F(1,15)<1$, n.s.], primary task $[F(1,15)=1.10$, n.s.], or interference task $[F(1,15)<1$, n.s.]. Importantly, the interaction be-
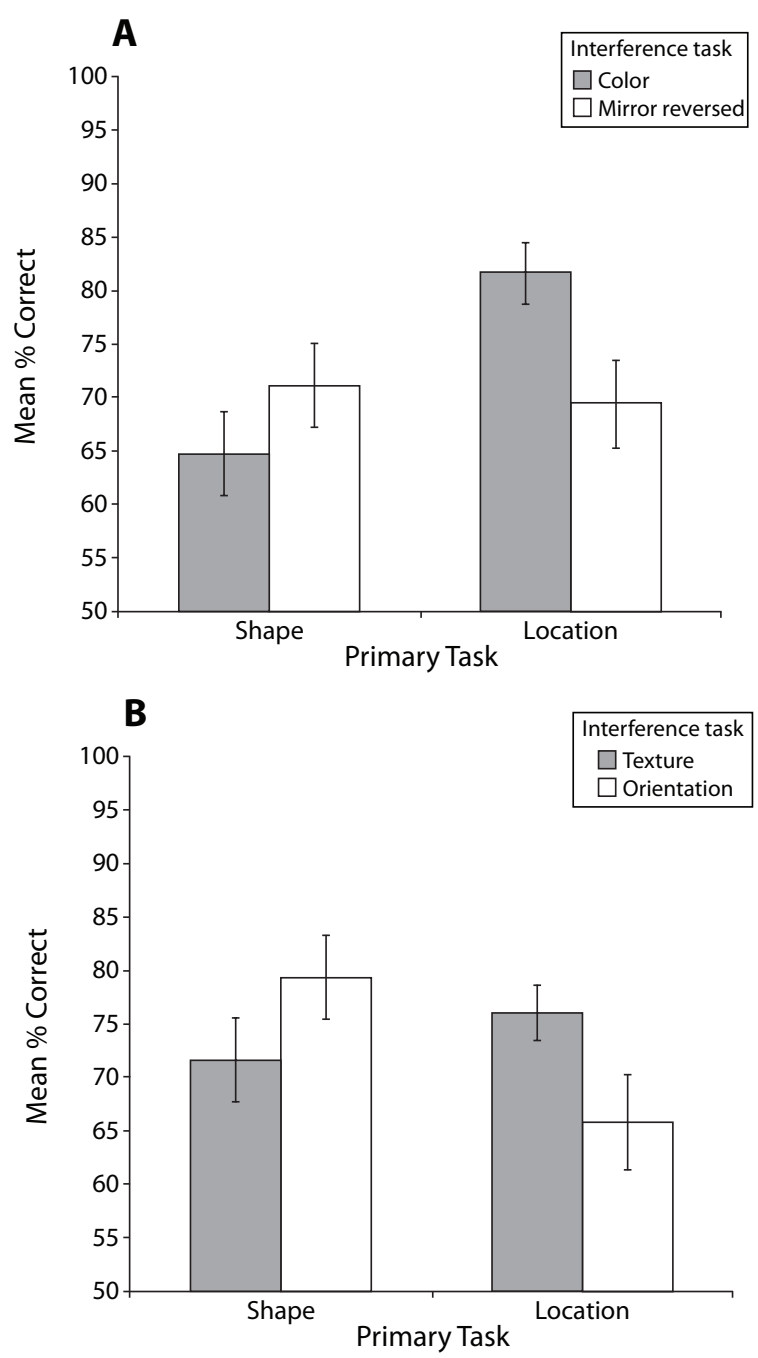

Figure 2. Illustration of the interaction between the primary tasks and the interference tasks for accuracy performance on the primary task when the interference tasks involved different stimuli (Experiment 1). (A) Interaction in the visual modality. (B) Interaction in the tactile modality.

tween primary task and interference task was significant $\left[F(1,15)=6.80, M S_{\mathrm{e}}=2,371.88, p<.05\right]$. This interaction is shown in Figures 2A and 2B for the visual and tactile modalities, respectively. We also found a significant interaction between modality and primary task $[F(1,15)=$ $\left.8.05, M S_{\mathrm{e}}=962.51, p<.05\right]$. There was no interaction between modality and interference task $[F(1,15)<1$, n.s. $]$, nor was there a three-way interaction between modality, primary task, and interference task $[F(1,15)<1$, n.s.].

A planned comparison post hoc analysis on the interaction between the primary and the interference tasks was performed. The difference in performance on the shapematching primary task between the shape and the location interference tasks approached significance $[F(1,15)=$ $4.28, p=.057]$. We found a significant difference in performance on the location primary task between the two 
interference tasks $[F(1,15)=6.38, p<.05]$. This analysis confirms that accuracy in each of the primary tasks was significantly better when the primary and the interference tasks involved different "what" and "where" resources but that performance was impaired when the tasks involved the same resources (e.g., shape and color matching).

We compared performance in the interference conditions with data collected from the 16 other participants who conducted the visual or haptic primary task alone, without interference. We wanted to establish whether or not interference had occurred in these conditions alone. Using unpaired $t$ tests, we found no significant difference between performance in the shape primary task alone and performance in the shape primary task with a location interference task in either the visual $[t(28)=1.86$, n.s. $]$ or the tactile $[t(28)=1.93$, n.s. $]$ domains. However, performance was significantly different between the shape primary task alone and the shape primary task with a shape interference task for both vision $[t(28)=3.04, p<$ $.01]$ and touch $[t(28)=3.71, p<.01]$, with primary task performance being worse with a shape interference task. Similarly, we found no significant difference in performance between the location primary task alone and the location primary task with a shape interference task in vision $[t(28)=-0.09$, n.s. $]$ or in touch $[t(28)=0.085$, n.s.]. However, performance was significantly different between the location primary task alone and the location primary task with a location interference task in vision $[t(28)=2.52, p<.05]$ and in touch $[t(28)=2.73, p<$ $.05]$, with primary task performance being worse with a location interference task.

As was mentioned above in the General Method section, the participants responded twice within each trial: first to the interference stimuli (S2 and $\mathrm{S} 3$ ) and then to the primary task stimuli (matching S1 to S4), which was the main dependent variable (see Figure 1 for an outline of the structure of a trial). To establish that the interference tasks were equal in terms of their difficulty, we compared the response data to the interference stimuli (i.e., matching performance between S2 and S3), using a one-way ANOVA on accuracy performance across the shape and location interference tasks only for each modality. There was no main effect of the visual interference task $[F(1,11)<1$, n.s. $]$ or of the tactile interference tasks $[F(1,15)<1$, n.s. $]$, indicating that changes in performance in the primary tasks were not due to varying task loads across the interference tasks.

\section{Discussion}

Our findings suggest that the resources for shape and location information are somewhat independent in both vision and touch. When the interference task recruited the same resources as the primary task (e.g., both "what" tasks, such as shape and color matching in vision), the participants had more difficulty retaining the stimulus properties of the primary task while performing the interference task. However, when the two tasks recruited putatively independent resources (such as a "where" or feature location task and a "what" texture-matching task in touch), memory for the stimulus properties of the primary task was affected less. In this case, the participants were able to more ef- ficiently retain the first stimulus of the primary task in memory while performing the interference task.

Previous literature suggests that for the visual system at least, information is processed independently according to recognition or spatial localization tasks (e.g., Tresch et al., 1993). Here, we extend this literature by presenting evidence that the tactile system is similarly organized. In the following experiments, we modified our paradigm to allow us to investigate the extent to which these resources are shared or are independent across modalities.

\section{EXPERIMENT 2A}

The two following experiments were designed to be preliminary to Experiment 3. Our main intention was to investigate the effects of task-dependent performance across modalities. However, in order to do this, we needed to establish an effective stimulus set that could be used as primary and interference stimuli across modalities. We were concerned that the interference stimuli used in the previous experiment would not be appropriate for crossmodal investigations, due to differences in the perceptual salience of stimulus types in different modalities. For example, some of our tasks were necessarily specific to one modality (i.e., color information cannot be encoded through touch, and the resolution for texture is greater in the tactile than in the visual system); consequently, these qualitative changes across interference tasks would not have been controlled. In order to avoid these concerns, we decided to use the same stimulus set interchangeably across modalities and across tasks; thus, the only relevant manipulation in the cross-modal experiment was the nature of the task performed (shape or location matching). First, however, we conducted two unimodal tasks in order to establish that the interference effects found in Experiment 1 were replicable even when the same stimulus set was used interchangeably in all conditions. Experiment 2A was conducted within the visual modality, and Experiment 2B was conducted within the tactile modality.

\section{Method}

Participants. Twenty-four participants ( 8 of them male and 16 female) from 15 to 34 years of age (mean age, 24 years) took part in this experiment; none had taken part in the previous experiment, and all reported normal or corrected-to-normal vision. All the participants reported being right-handed. Each participant provided written consent before the experiment began.

Stimuli. The same visual stimuli were used interchangeably between the shape and the location tasks, irrespective of whether they were primary or interference tasks (see the General Method section for further details).

Design. The experiment was based on a repeated measures $2 \times 2$ design with primary task (shape or location) and interference task (shape or location) as factors. Thus, there were four different blocks, each consisting of one of the possible pairings of shape and location primary tasks with shape and location interference tasks. The order of the experimental blocks was counterbalanced across participants. The participants completed all four experimental blocks.

Procedure. The participants were instructed in advance of each of the four experimental blocks as to the nature of the primary and interference tasks in the trials. There was a $2,000-\mathrm{msec}$ ITI and a $1,000-\mathrm{msec}$ ISI within a trial. The stimuli were presented for a duration of $55 \mathrm{msec}$ in the shape condition and $220 \mathrm{msec}$ in the location 
condition. These presentation times were established in a previous pilot study (with a different group of participants), which allowed us to compare performance and adjust timings until performance was equivalent across both the shape and the location tasks. There were 80 trials in total, with 20 trials in each block. The experiment lasted approximately $30 \mathrm{~min}$.

\section{Results}

Because overall performance was higher for all the participants in this task than for those in the previous task, we set a response criterion of $70 \%$ for the interference tasks to ensure that the participants were performing this task. Data from 5 participants were excluded from analysis because their performance was below this criterion in at least one block, even after practice.

A $2 \times 2$ repeated measures ANOVA with the factors of primary task (shape or location) and interference task (shape or location) was performed on the accuracy data. There was no main effect of primary task $[F(1,18)=$ 1.09 , n.s.], nor was there a main effect of interference task $[F(1,18)<1$, n.s.]. Since performance was the same across the primary tasks and the interference tasks, we were satisfied that these tasks were equally difficult. More interesting, we found a significant interaction between these two factors $\left[F(1,18)=9.89, M S_{\mathrm{e}}=93.457, p<\right.$ $.01]$. As is shown in Figure 3, accuracy performance on the shape-matching primary task was reduced by a shape interference task (mean shape primary task accuracy was $69.7 \%$ ), relative to when the location interference task was presented (mean shape primary task accuracy was $75.5 \%$ ). Conversely, performance on the location primary task was reduced by a location interference task (mean location primary task accuracy was $65.5 \%$ ), relative to when

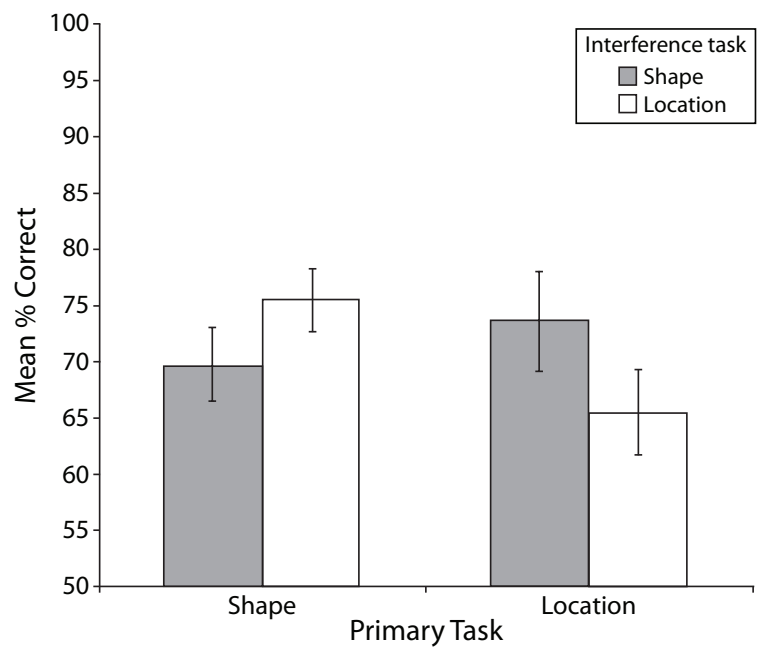

Figure 3. Interaction between the primary tasks and the interference tasks for accuracy performance on the primary task for the visual modality only (Experiment $2 \mathrm{~A}$ ). For a primary shape-matching task ("what"), performance was significantly reduced by a shape-matching interference task, relative to a location-matching interference task. Conversely, performance on a location-matching primary task was reduced by a locationmatching interference task, relative to a shape-matching interference task. the shape interference task was presented (mean location primary task accuracy was $73.6 \%$ ).

A planned comparison post hoc analysis was conducted to further examine this interaction. There was a significant difference in performance on the shape primary task between the shape and the location interference tasks $[F(1,18)=4.96, p<.05]$. Accuracy on the shape primary task was significantly better when the interference task was location, relative to when the interference task was shape. There was also a significant difference between performance on the location primary task and that on the two interference tasks $[F(1,18)=5.61, p<.05]$. Accuracy in the location primary task was significantly better when the interference task was shape matching, relative to when the interference task was location matching. Therefore, accuracy on each of the primary tasks was significantly better when the primary and interference tasks were different, suggesting that processing in each of the shape and location systems is relatively independent.

We compared performance in all the primary and interference task conditions against data collected from 16 other participants who conducted the primary task alone, without interference. For these participants, performance on the shape primary task was $79.33 \%$ and on the location primary task was $82.67 \%$. Using unpaired $t$ tests, we found no significant difference between performance on the shape primary task alone and performance on the shape primary task with a location interference task $[t(37)=0.73$, n.s. $]$. However, performance was significantly different between that on the shape primary task alone and that on the shape primary task with a shape interference task $[t(37)=2.01, p<.05]$, with primary task performance being worse when a shape interference task was introduced. We found no significant difference between performance on the location primary task alone and performance on the location primary task with a shape interference task $[t(37)=1.80, p=.08]$. Again, there was a significant difference between performance on the location primary task alone and that on the location primary task with a location interference task $[t(37)=3.59, p<$ $.01]$, with primary task performance being worse when a location interference task was introduced. These findings suggest that interference effects were specific to those conditions in which the primary and the interference tasks were the same.

As in Experiment 1, a one-way repeated measures ANOVA was conducted for the performance on the interference tasks only. We found no difference between performance on the shape and location interference tasks $[F(1,18)=1.26$, n.s.], suggesting that there was no difference in cognitive load between the tasks. In other words, performance on the primary task was not due to varying task loads across the interference tasks.

\section{Discussion}

Our data here provide further behavioral evidence for dissociated shape and location visual processing when the same stimuli are used in both tasks. An interference task decreased accuracy in the primary task when the two tasks were the same (e.g., two shape tasks), relative to when 
they were different (e.g., a primary shape task and an interference location task), even though the same stimuli were used throughout.

Our findings support the neuropsychological and neuroimaging evidence for independent processing of identification and spatial tasks, and they replicate the findings of previous behavioral studies (e.g., Logie \& Marchetti, 1991; Tresch et al., 1993). Having demonstrated the effects of separate processing of shape and location information on performance within the visual domain, our next aim was to replicate the findings in Experiment 1 with haptic processing, using the same stimuli interchangeably across tasks.

\section{EXPERIMENT 2B}

We expected to find the same pattern of results as that found in Experiment 1-that is, that accuracy in the primary task should decrease when the interference task was the same, relative to when the two tasks were different.

\section{Method}

Participants. Twenty-four participants (12 of them male and 12 female) from 19 to 47 years of age (mean age, 27 years) took part in this experiment. All the participants reported normal or correctedto-normal vision and no haptic impairments. Twenty-two participants reported being right-handed, and 2 reported being left-hand dominant. Written consent was provided by each participant before the experiment began. None of these participants had taken part in the previous experiments.

Design. The design was the same as that in Experiment 1.

Procedure. The procedure in this experiment was the same as that in the previous experiments, with the following exceptions. Here, the stimuli were 3-D models of the visual stimuli used in Experiment 2A (see the General Method section). Haptic stimuli were presented for 4,000 msec in the shape condition and for 3,000 msec in the location condition. The presentation times were determined in a previous pilot study (with a different group of participants), which allowed us to compare performance and adjust timings until performance was equivalent across the shape and location tasks.

Accuracy rates were recorded for both the primary and the interference tasks to ensure that the participants were performing the task as instructed. There were four blocks in this experiment, with 10 trials in each block. Block order was counterbalanced across participants. The experiment took around $60 \mathrm{~min}$ to complete.

\section{Results}

As in Experiment 1, we wanted to ensure that the participants were performing the interference task in all blocks; consequently, all the participants who scored below a criterion response of $70 \%$ correct for the interference task (adopted from Experiment 2A) in any block were excluded. As a result, data from 12 participants were excluded from analysis, since these participants failed to reach criterion performance in at least one block, even after extensive practice. ${ }^{2}$ We conducted a $2 \times 2$ repeated measures ANOVA of the accuracy performance, with primary task (shape or location) and interference task (shape or location) as factors. There was no main effect of primary task $[F(1,11)<1$, n.s.] or of interference task $[F(1,11)=2.44$, n.s.]. We were therefore assured that the level of difficulty between the shape and the location tasks was equivalent. As was predicted, we found an interac- tion between the primary and the interference task factors $\left[F(1,11)=17.32, M S_{\mathrm{e}}=192.42, p<.01\right]$. As is shown in Figure 4 , accuracy in the primary task was reduced by the interference task when both were the same. Performance in the shape primary task was reduced by the shape interference task, relative to the location interference task (the mean shape primary task accuracy was reduced from $84.2 \%$ to $72.5 \%$ with shape interference). Conversely, performance in a location primary task was reduced by a location interference task, relative to a shape interference task (i.e., mean location primary task accuracy was reduced from $87.5 \%$ to $65.8 \%$ ).

A planned comparison post hoc analysis on the interaction between the primary and the interference tasks was performed. There was a significant difference in performance on the shape matching primary task between the shape and the location interference tasks $[F(1,11)=4.96$, $p<.05]$. There was also a significant difference in performance on the location primary task between the two interference tasks $[F(1,11)=17.37, p<.01]$. This analysis confirms that accuracy in each of the primary tasks was significantly better when the primary and the interference tasks were different, as compared with when the tasks were the same.

We compared performance in the conditions in which the tasks were different with data collected from 16 other participants who conducted the haptic primary task alone, without interference. Performance on the shape task alone was $89.33 \%$, and on the location task it was $86.67 \%$. Using unpaired $t$ tests, we found no significant difference between shape primary task alone performance and shape primary task performance with a location interference task $[t(31)=1.00$, n.s. $]$. However, we did find a significant difference between shape primary task alone performance and shape primary task performance with a shape interference task $[t(31)=3.34, p<.01]$, with primary task performance being worse when a shape interference task

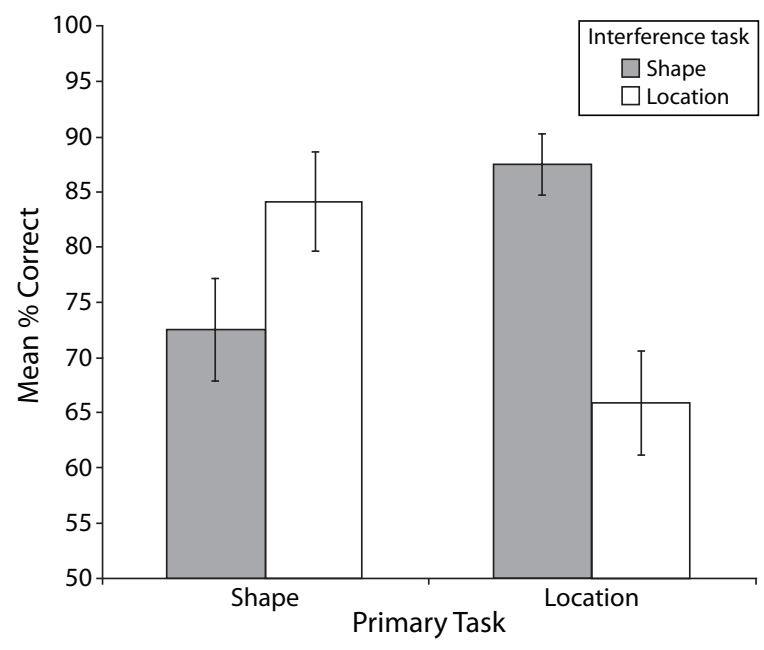

Figure 4. Interaction between the primary tasks and the interference tasks for accuracy performance on the primary task in the tactile modality only (Experiment 2B). 
was introduced. Similarly, we found no significant difference between location primary task alone performance and location primary task performance with a shape interference task $[t(31)=-0.57$, n.s. $]$. However, we did find a significant difference between location primary task alone performance and location primary task performance with a location interference task $[t(31)=3.98, p<.01]$, with primary task performance being worse when a location interference task was introduced.

As in Experiments 1 and 2A, we performed a one-way ANOVA for accuracy performance across the shape and location interference tasks only (i.e., the responses to matching S2 and S3, as shown in Figure 1). There was no main effect of the interference task $[F(1,11)<1$, n.s.], suggesting that changes in performance on the primary tasks were not due to varying task loads across the interference tasks.

\section{Discussion}

The results of Experiment 2B provide further behavioral evidence that the haptic system in humans may use the same functional principles of information processing as the visual system. As in Experiments 1 and 2A, task interference was reduced when the primary and the interference tasks were different, suggesting that shape and location processing are relatively independent, as they are in vision.

We conducted an informal observation of the way in which the participants explored the stimuli during both shape and location tasks in order to see whether stimulus exploration was task specific even though the stimuli were the same. Lederman and Klatzky (1987) have argued that different exploratory procedures (EPs) are employed depending on the nature of the task; therefore, we expected that the participants would adopt different EPs across the shape and location tasks. This is indeed what we found. We observed that the participants used a contour-tracing EP during the shape-matching tasks, whereas during the location-matching tasks the participants used a lateral motion EP of the hand across the object's surface. Lateral motion of the hand is generally used for haptic texture perception, as opposed to shape perception. Although this observation was informal, task-dependent EPs were consistently observed across participants; therefore, we were convinced that our tasks involved different types of information processing of the same set of stimuli.

\section{EXPERIMENT 3}

Our previous experiments were designed not only to test the idea that resources within each of the visual and haptic systems were task dependent, but also to establish a paradigm that would allow us to investigate taskdependent resources across these modalities. Neuroimaging evidence suggests that the same cortical areas are activated for shape processing across vision and touch. Specifically, the lateral occipital complex has been implicated in both visual and haptic object recognition for both familiar objects (Amedi, Malach, Hendler, Peled, \& Zohary, 2001; Reed, Shoham, \& Halgren, 2004) and novel shapes (James et al., 2002). These studies have suggested that information, at least for the purpose of identification, may be modality independent. Evidence for the same cortical substrates underlying cross-modal spatial perception in humans is, however, less forthcoming, although recent studies suggest that visual cortical areas, such as the superior parietal cortex (Reed et al., 2005) and right intraparietal sulcus (Kitada et al., 2006), are recruited for haptic spatial tasks.

The aim of the present experiment was to extend the within-modality findings to investigate whether taskdependent resources generalize across modalities. We predicted that if resources are specific to each modality, there would be no interference across modalities. On the other hand, if resources are shared, as has been suggested by previous literature, we predicted that task-dependent, cross-modal interference would take place.

\section{Method}

Participants. Twenty-four participants ( 9 of them male and 15 female) from 15 to 37 years of age (mean age, 20 years) took part in this experiment. All the participants reported normal or correctedto-normal vision, and none reported any haptic impairment. One of the participants reported being left-hand dominant, and all others reported being right-handed. Written consent was provided by each participant before the experiment began. None of these participants had been involved in the previous experiments.

Apparatus and Stimuli. See the General Method section for a description.

Design. The experiment was based on a three-way repeated measures design with sensory modality of the primary task (vision or touch), primary task (shape or location), and interference task (shape or location) as factors. The experiment was divided into two main experimental blocks, each based on the sensory modality of the primary task, with four blocks of primary and interference task combinations in each. The interference task was always conducted in a modality different from that of the primary task. There were 10 trials in each block. The order of the trials was randomized within each block, and the block order was counterbalanced across participants.

Procedure. The procedure was mainly the same as those in the previous experiments, except that here, the interference task was conducted in a modality different from that of the primary task. The stimulus presentation timings were the same as those in Experiments $2 \mathrm{~A}$ and $2 \mathrm{~B}$ according to the modality. Thus, stimulus presentation durations for the haptic and visual modalities were 4,000 and $55 \mathrm{msec}$, respectively, for shape matching and 3,000 and $220 \mathrm{msec}$, respectively, for the location tasks. As in the previous experiments, auditory "clicks" signaled the participant to begin or end feeling the stimulus.

We included an ITI of $25 \mathrm{sec}$ in order to allow the experimenter sufficient time to replace the haptic stimuli from the previous trial with the stimuli in the upcoming trial. There was a 5 -sec ISI between haptic stimuli (to allow the experimenter time to switch the stimuli) and a 1-sec ISI between presentations of visual stimuli (as in the previous experiments). Each block began with four practice trials, which were repeated if necessary. The participants were informed prior to each block in which modality the primary and interference tasks would be conducted. On average, the experiment took $75 \mathrm{~min}$ to complete for each participant.

\section{Results}

A $70 \%$ accuracy response criterion was set for the interference task in each block. The participants who did not achieve this level of performance across all blocks were excluded from analysis, since a drop in performance indicated that the participant was not performing the task. 
Consequently, data from 6 participants were excluded from analysis because their performance was below the $70 \%$ criterion even after extensive practice.

We conducted a three-way repeated measures ANOVA with sensory modality of the primary task (vision or touch), primary task (shape or location), and interference task (shape or location) as factors. We found a main effect of sensory modality $\left[F(1,17)=11.37, M S_{\mathrm{e}}=\right.$ $206.34, p<.05]$, in that performance on the haptic primary tasks $(85.55 \%$ accuracy) was significantly better than that on the visual primary tasks ( $77.48 \%$ accuracy). We also found a significant main effect of primary task $\left[F(1,17)=6.24, M S_{\mathrm{e}}=331.42, p<.05\right]$, where performance on the location-matching primary task $(85.31 \%$ accuracy) was significantly better than performance on the shape-matching primary task $(77.73 \%$ accuracy). There was no main effect of the interference task $[F(1,17)<1$, n.s.]. More pertinently, we found a significant interaction between the primary and the interference tasks $[F(1,17)=$ $\left.5.45, M S_{\mathrm{e}}=142.61, p<.05\right]$. This interaction is plotted in Figure 5. ${ }^{3}$ There was no interaction between the factors of sensory modality and primary task $[F(1,17)<1$, n.s.] or between sensory modality and interference task $[F(1,17)=2.76$, n.s.]. We found no evidence of a threeway interaction between the factors of sensory modality, primary task, and interference task $[F(1,17)=1.41$, n.s.], suggesting that the effects of interference occurred in both sensory modalities.

A planned comparison post hoc analysis was conducted on the interaction between the primary and the interference task factors. We found no difference between the shape and location interference tasks for performance on the shape primary task $[F(1,17)=1.36$, n.s.]. On the other hand, performance on the location primary task was significantly reduced by the location interference task, relative to the shape interference task $[F(1,17)=5.41$, $p<.05]$. Moreover, when the interference task was a

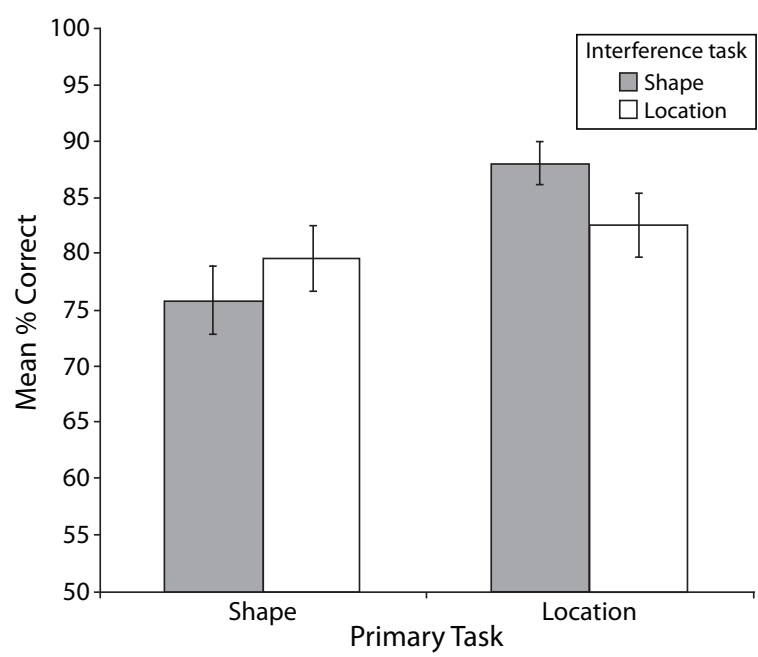

Figure 5. Interaction between the primary tasks and the interference tasks on accuracy performance in the primary task, collapsed across modalities (Experiment 3 ). shape task, this resulted in performance that was worse in the shape primary task than in the location primary task $[F(1,17)=10.22, p<.05]$. A location interference task, on the other hand, had the same effect on performance on both the shape and the location primary tasks $[F<1$, n.s.]. Since performance on the shape primary task was, overall, worse than that on the location primary task and both shape and location interference tasks had the same effect on performance on the shape primary task, these data suggest that both cross-modal shape and location affected primary shape task performance. Conversely, only the cross-modal location task affected location primary task performance but not shape primary task performance.

As in the previous experiments, we compared performance in conditions when the primary and interference tasks were the same with the average performance from the group of 16 participants who conducted the primary tasks alone. Using unpaired $t$ tests, we found no significant difference between performance on the primary shape task alone and that on a shape primary task with a location interference task $[t(37)=1.33$, n.s.]. Performance when the primary and interference tasks were both shape tasks was significantly worse than performance on the primary shape task alone $[t(37)=2.32, p<.05]$. When both the primary and the interference tasks were location tasks, performance in this condition was not significantly different from performance on the primary location task alone $[t(37)=0.125$, n.s.]. There was also no significant difference between performance on the primary location task alone and performance on the location primary task with a shape interference task $[t(37)=-1.16$, n.s. $]$.

These findings, together with the source of the interaction between the primary and interference tasks in this experiment, suggest that resources for shape are shared across modalities and that resources for location processing are also, to some extent, shared by shape resources across these modalities. Location processing in one modality, on the other hand, seems to be affected by similar processing in the other, suggesting that resources for spatial location, like resources for shape processing, are modality independent. Unlike performance in the shape primary task condition, a shape interference task did not affect location processing, suggesting an interesting asymmetry of overlapping resources between locationand shape-based tasks.

Finally, we analyzed the responses to the interference task only (S2 and S3) to ensure that the participants were performing the task equally well across modalities. We found no effect of sensory modality $[F(1,17)<1$, n.s.] or of interference task $[F(1,17)<1$, n.s.], suggesting that there was no difference in difficulty either across the shape and location interference tasks or across the modalities. In other words, performance on the primary task was not due to varying task loads across the interference tasks, regardless of modality.

\section{Discussion}

Our findings suggest that tasks conducted in one modality can affect performance in another modality. Thus, even though performance overall was better in the haptic primary 
task, the interaction between primary and interference tasks suggests that interference occurred across sensory modalities. Furthermore, especially for location processing, crossmodal interference seems to be task specific.

As in our previous experiments, we found an interaction between performance on the primary and interference tasks, even though these tasks were presented across different modalities. On initial analysis, this finding suggests that resources across modalities are task dependent. However, on further inspection of the interaction, it was revealed that there was no difference between the interference tasks on the primary shape task (see Figure 5). In other words, it seems that the interference observed for performance within the shape primary task occurred from both a cross-modal shape and a cross-modal spatial location task. Thus, our findings suggest not only that resources for shape processing are shared across modalities, but also that some aspect of spatial processing is also involved in shape perception across vision and touch. This finding may be specific to localization tasks based on small-scale objects, and it is a matter for future research whether such resources are shared for localization in larger scale scenes.

Performance on the location primary task, on the other hand, was significantly reduced by a cross-modal location, relative to a shape interference task. Moreover, performance on the location primary task when the interference task was a shape task was not different from performance on the location task alone. This finding suggests that shape processing did not interfere with location processing across modalities.

One concern we had about the difference in these tasks was that although we used the same stimulus presentation times and ISIs as in our previous experiments, different delays across conditions resulted. For example, when the interference task was haptic, the interval between the primary visual stimuli (S1 and S4) was between 13 and $15 \mathrm{sec}$. On the other hand, when the interference task was visual, the interval between haptic S1 and S4 was approximately 7,000 msec. Thus, visual primary stimuli needed to be retained for a longer time than haptic primary stimuli. This differential delay between primary task stimuli across the modalities may have caused the main effect of modality. Furthermore, we were concerned that these differences in intervals across the conditions may have contributed to the interaction between the primary and the interference tasks. To test whether our findings were robust, we repeated this experiment with another 16 naive participants, but we used the same interval time of $15 \mathrm{sec}$ between $\mathrm{S} 1$ and S4, irrespective of modality and task. The presentation times of the stimuli were the same as those in the previous experiment. Again, we found a significant effect of modality, with performance in the haptic modality being significantly better than that in the visual $\left[F(1,15)=16.22, M S_{\mathrm{e}}=209.79, p<.01\right]$. We did not, however, find a main effect of primary task $[F(1,15)=$ 1.18 , n.s.] or of interference task $[F(1,15)<1$, n.s.]. There was a significant interaction between the primary and the interference tasks $\left[F(1,15)=5.42, M S_{\mathrm{e}}=129.79\right.$, $p<.05]$. No other interactions between the factors were found. On the basis of this new finding, we were assured that the effects of the interference tasks on the primary tasks were robust to changes in the intervals between the primary task stimuli.

\section{GENERAL DISCUSSION}

The results of Experiments 1 and 2A provide further evidence that the processing of visual information for recognition (i.e., "what"-type processing) is independent of the processing of information for spatial localization (i.e., "where"-type processing). These findings extend those in the previous literature by providing evidence that "what" and "where" processing are also independent in the human touch system (Experiments 1 and 2B). Moreover, we confirmed that our results are a reflection of how information is processed according to the type of task, since task-specific effects were independent of the type of stimuli used for the interference task, relative to those in the primary task. In order to maintain the same levels of task difficulty and stimulus discriminability in our study for the cross-modal task, it was necessary to use the same set of stimuli for both the "what" and the "where" tasks interchangeably to avoid effects due to cognitive or attentional demands. We investigated whether or not taskdependent processing occurs only within each of these modalities and, instead, found that resources for both shape and spatial tasks are shared across vision and touch (Experiment 3). Moreover, some components of spatial resources are also shared by resources for shape perception across these modalities. In summary, therefore, we found behavioral evidence for a double dissociation of interference between both shape-based and location-based tasks within and across the visual and touch modalities.

In Experiment 1, we found evidence that an interference task associated with "what" or "where" processing (see, e.g., Ungerleider \& Mishkin, 1982) selectively interfered with performance in the shape or location task, respectively. Specifically, we investigated whether color processing in our visual tasks or texture processing in our tactile tasks (i.e., putatively ventral tasks) would interfere with shape recognition in each modality. Furthermore, we investigated whether image rotation matching or orientation of textured stimuli would interfere with a feature localization task in the visual or tactile domain, respectively. We expected interference effects within "what" and "where" systems, but not across these systems. For vision, we found that a color-matching task embedded in a primary shape-matching task resulted in a cost in performance, relative to when it was embedded in a feature localization task. In the tactile domain, matching the orientation of patches of sandpaper affected a cost in performance in a feature-location-matching task, but not in a shapematching task. These results strongly suggest that information is processed in a manner that is based on whether the task is one of object recognition or spatial perception, rather than the similarity of the tasks themselves.

Our results suggest that the manners in which information for recognition and spatial location is processed by touch and by vision are similar. Our findings within the 
touch domain complement those from the neuropsychological and neuroimaging literatures (e.g., Prather, Votaw, \& Sathian, 2004; Reed \& Caselli, 1994; Reed et al., 1996; Reed et al., 2005) and, taken together, suggest a separation between shape and location processing at a functional level within the haptic system. It is interesting to note, however, that many neuroimaging studies have reported activation of what are considered visual cortical areas during haptic processing (e.g., Amedi et al., 2001; James et al., 2002; Kitada et al., 2006), raising the question about what mediates haptic shape and spatial perception. Prather et al., for example, argued that visual imagery mediates tactile processing. It is possible, therefore, that the findings from the haptic tasks reflect a transfer of information to visual imagery. If this were the case, then, contrary to our findings, performance within the visual domain would be expected to be at least as good as performance in the haptic domain (see Experiment 3). Other findings reported by Reed et al. (2004) suggest that haptic processing activates cortical areas not generally implicated in visual processing, suggesting that visual imagery does not necessarily mediate haptic perception. Whatever the case, it is beyond the scope of the present study to speculate on how information processing occurs across the visual and haptic domains. Suffice it to say that the results from Experiment 3 suggest that resources are shared across modalities and that these findings may reflect a transfer of some information from touch to vision.

It is interesting to note that for the majority of the participants, the interference tasks in these experiments did not reduce primary task performance to chance level but, instead, caused only relative interference, as compared with when the primary and the interference tasks were different. Complete interference might be expected, for example, because of the high degree of interstimulus and intertask similarity, especially in Experiments 2 and 3. Moreover, Tresch et al. (1993) reported evidence of complete interference, so that primary task performance was reduced to chance levels. However, interference effects in Experiment 1, where we adopted a paradigm similar to that in Tresch et al., also did not render performance close to chance level. Although the difference between our findings and those in the Tresch et al. study could be due to subtle differences in the paradigms between these studies, the question nevertheless remains as to why we did not find total interference when the two tasks were the same. We can suggest two possible reasons as to why this might have been the case. First, it may be that some non-shape- or location-specific information was encoded (e.g., verbal descriptions) that may not have been vulnerable to perceptual interference. Second, the delay in presentation time between the matching stimuli may have resulted in different memory representations of these stimuli. For example, the first stimulus may have been committed to long-term memory, whereas the second, matching stimulus was probably processed in working memory alone. These differences in representations may have prevented complete interference from occurring across tasks. Clearly, these suggestions require further investigation, and neuroimaging techniques may tease apart the different possibilities.

We made every effort to ensure that our results could not be due to varying attentional or extraneous cognitive loads across the tasks. We were confident that this was the case, since performance either between the shape and the location interference tasks or between both primary tasks, at least within the modalities, was not significantly different (see Experiments 1, 2A, and 2B). Furthermore, pilot studies were conducted to match performance between these two tasks, which was achieved by manipulating different presentation durations across tasks. Performance in the cross-modal task (Experiment 3), however, was better in the location than in the shape task. Our data suggest that this main effect was mediated by a greater effect of interference in the shape primary task than in the location primary task. That is, both shape and location interference tasks affected performance in the shape primary task. The fact that there was no main effect of interference task suggests that these tasks were equally difficult but that their interference effect was dependent on the primary tasks.

According to the findings in Experiment 3, the processing of object information for recognition or for spatial information is independent of modality. The overall interaction between primary task performance and interference task performance in the cross-modal task (Experiment 3 ) is consistent with that in the unimodal experiments (Experiments 1, 2A, and 2B). However, unlike in our previous experiments, we found no difference between the interference tasks for performance on the shape-processing primary task. This finding suggests one of two possibilities. First, it is possible that multisensory shape perception involves a network of resources, some of which are also involved in spatial perception. Indeed, preliminary findings from a neuroimaging study suggest this to be the case (Calvert, Hansen, Steven, \& Newell, 2007). On the other hand, the effect of spatial interference on cross-modal shape tasks may have occurred indirectly or may have been mediated through another sensory modality. For example, the results of Smyth and Pendleton (1989) and from Kerzel (2001) suggest than kinesthetic information, such as hand movements from one location to another, can interfere with the memory for visual and haptic events. This suggests the possibility that the hand movements involved in the spatial tasks may have interfered with the memory for the shape of an object, irrespective of modality. This is probably unlikely, however, since hand movements were involved in both shape and spatial tasks and the scales of these movements were similar. Moreover, kinesthetic spatial information has not been found to interfere with memory for identity (Smyth \& Pendleton, 1989). However, differences between the task-dependent EPs may account for changes in kinesthetic information that may have an effect on the processing of object shape; therefore, one cannot rule it out as a possibility. Clearly, further research is required to investigate to what extent the network of resources for shape recognition and spatial localization tasks are shared across the modalities. 


\section{AUTHOR NOTE}

This study was funded by the European Commission under "Information Society Technologies" Program Grant IST-2001-34712 and by the Irish Research Council for the Humanities and Social Sciences. Correspondence concerning this article should be addressed to F. N. Newell, School of Psychology, Trinity College, Dublin 2, Ireland (e-mail: fiona .newell@tcd.ie).

\section{REFERENCES}

Amedi, A., Malach, R., Hendler, T., Peled, S., \& Zohary, E. (2001). Visuo-haptic, object-related activation in the ventral visual pathway. Nature Neuroscience, 4, 324-330.

Avillac, M., Denève, S., Olivier, E., Pouget, A., \& Duhamel, J. R. (2005). Reference frames for representing visual and tactile locations in parietal cortex. Nature Neuroscience, 8, 941-949.

Belin, P., \& Zatorre, R. J. (2000). "What," "where" and "how" in auditory cortex. Nature Neuroscience, 3, 965-966.

Calvert, G. A., Hansen, P. C., Steven, M. S., \& Newell, F. N. (2007). An fMRI comparative study of visuotactile "what" and "where" systems. Manuscript in preparation.

Clarke, S., Thiran, A. B., Maeder, P., Adriani, M., Vernet, O., RegLi, L., ET AL. (2002). What and where in human audition: Selective deficits following focal hemispheric lesions. Experimental Brain Research, 147, 8-15.

Desimone, R., Schein, S. J., Moran, J., \& Ungerleider, L. G. (1985). Contour, color and shape analysis beyond the striate cortex. Vision Research, 25, 441-452.

Desimone, R., \& Ungerleider, L. G. (1986). Multiple visual areas in the caudal superior temporal sulcus of the macaque. Journal of Comparative Neurology, 248, 164-189.

Duncan, J. (1984). Selective attention and the organization of visual information. Journal of Experimental Psychology: General, 113, 501-517.

Easton, R. D., Srinivas, K., \& Greene, A. J. (1997). Do vision and haptics share common representations? Implicit and explicit memory within and between modalities. Journal of Experimental Psychology: Learning, Memory, \& Cognition, 23, 153-163.

Egly, R., Driver, J., \& Rafal, R. D. (1994). Shifting visual attention between objects and locations: Evidence from normal and parietal lesion subjects. Journal of Experimental Psychology: General, 123, 161-177.

Felleman, D. J., \& Van Essen, D. C. (1991). Distributed hierarchical processing in the primate cerebral cortex. Cerebral Cortex, 1, 1-47.

Goodale, M. A., \& Milner, A. D. (1992). Separate visual pathways for perception and action. Trends in Neurosciences, 15, 20-25.

Haxby, J. V., Grady, C. L., Horwitz, B., Ungerleider, L. G., MishKIn, M., Carson, R. E., ET AL. (1991). Dissociation of object and spatial visual processing pathways in human extrastriate cortex. Proceedings of the National Academy of Sciences, 88, 1621-1625.

Haxby, J. V., Horwitz, B., Ungerleider, L. G., Maisog, J. M., PieTRINI, P., \& GRADY, C. L. (1994). The functional organization of human extrastriate cortex: A PET-rCBF study of selective attention to faces and locations. Journal of Neuroscience, 14, 6336-6353.

Hirst, W., \& Kalmar, D. (1987). Characterizing attentional resources. Journal of Experimental Psychology: General, 116, 68-81.

Humphreys, G. W., \& RidDoch, M. J. (2003). From what to where: Neuropsychological evidence for implicit interactions between objectand space-based attention. Psychological Science, 14, 487-492.

Irwin, D. E., \& BRockmole, J. R. (2004). Suppressing where but not what: The effect of saccades on dorsal- and ventral-stream visual processing. Psychological Science, 15, 467-473.

James, T. W., Culham, J., Humphrey, G. K., Milner, A. D., \& Goodale, M. A. (2003). Ventral occipital lesions impair object recognition but not object-directed grasping: An fMRI study. Brain, 126, 2463-2475.

James, T. W., Humphrey, G. K., Gati, J. S., Servos, P., Menon, R. S., \& Goodale, M. A. (2002). Haptic study of three-dimensional objects activates extrastriate visual areas. Neuropsychologia, 40, 1706-1714.

KAPPERS, A. M. L. (1999). Large systematic deviations in the haptic perception of parellelity. Perception, 28, 1001-1012.

KerzeL, D. (2001). Visual short-term memory is influenced by haptic perception. Journal of Experimental Psychology: Learning, Memory, \& Cognition, 27, 1101-1109.

Kinsbourne, M. (1980). Mapping a behavioral cerebral space. International Journal of Neuroscience, 11, 45-50.

Kitada, R., Kito, T., Saito, D. N., Kochiyama, T., Matsumura, M., SadATo, N., \& Lederman, S. J. (2006). Multisensory activation of the intraparietal area when classifying grating orientation: A functional magnetic resonance imaging study. Journal of Neuroscience, 26, 7491-7501.

Landau, B., Hoffman, J. E., \& Kurz, N. (2006). Object recognition with severe spatial deficits in Williams syndrome: Sparing and breakdown. Cognition, 100, 483-510.

LEDERMAN, S. J., \& KLATZKY, R. J. (1987). Hand movements: A window into haptic object recognition. Cognitive Psychology, 19, 342-368.

Liu, T., Slotnick, S. D., Serences, J. T., \& Yantis, S. (2003). Cortical mechanisms of feature-based attentional control. Cerebral Cortex, 13, 1334-1343.

Logie, R. H., \& MARChetTi, C. (1991). Visuo-spatial working memory: Visual, spatial or central executive? In R. H. Logie \& M. Denis (Eds.), Mental images in human cognition (pp. 105-115). Amsterdam: NorthHolland.

Marois, R., Leung, H.-C., \& Gore, J. (2000). A stimulus-driven approach to object identity and location processing in the human brain. Neuron, 25, 717-728.

Merigan, W. H., \& Maunsell, J. H. (1993). How parallel are the primate visual pathways? Annual Review of Neuroscience, 16, 369-402.

Milner, D. A., \& Goodale, M. A. (1995). The visual brain in action. New York: Oxford University Press.

Mishim, M., Ungerleider, L. G., \& Macko, K. A. (1983). Object vision and spatial vision: Two cortical pathways. Trends in Neurosciences, 6, 414-417.

Newcombe, F., \& Russell, W. R. (1969). Dissociated visual perceptual and spatial deficits in focal lesions of the right hemisphere. Journal of Neurology, Neurosurgery, \& Psychiatry, 32, 73-81.

Newell, F. N., Ernst, M. O., Tuan, B. S., \& Bülthoff, H. H. (2001). Viewpoint dependence in visual and haptic object recognition. Psychological Science, 12, 37-42.

Newell, F. N., Woods, A. T., Mernagh, M., \& Bülthoff, H. H. (2005). Visual, haptic and crossmodal recognition of scenes. Experimental Brain Research, 161, 233-242.

Poremba, A., Saunders, R. C., Crane, A. M., Cook, M., Sokoloff, L., $\&$ Mishkin, M. (2003). Functional mapping of the primate auditory system. Science, 299, 568-572.

Prather, S. C., Votaw, J. R., \& Sathian, K. (2004). Task-specific recruitment of dorsal and ventral visual areas during tactile perception. Neuropsychologia, 42, 1079-1087.

ReAles, J. M., \& BALlesteros, S. (1999). Implicit and explicit memory for visual and haptic objects: Cross-modal priming depends on structural descriptions. Journal of Experimental Psychology: Learning, Memory, \& Cognition, 25, 644-663.

ReEd, C. L., \& CAselli, R. J. (1994). The nature of tactile agnosia: A case study. Neuropsychologia, 32, 527-539.

Reed, C. L., Caselli, R. J., \& Farah, M. J. (1996). Tactile agnosia: Underlying impairment and implications for normal tactile object recognition. Brain, 119, 875-888.

Reed, C. L., Klatzky, R. L., \& Halgren, E. (2005). What vs. where in touch: An fMRI study. NeuroImage, 25, 718-726.

Reed, C. L., Shoham, S., \& Halgren, E. (2004). Neural substrates of tactile object recognition: An fMRI study. Human Brain Mapping, 21, 236-246.

Romanski, L. M., Tian, B., Fritz, J., Mishrin, M., GoldmanRAKIC, P. S., \& RAUSCHECKER, J. P. (1999). Dual streams of auditory afferents target multiple domains in the primate prefrontal cortex. $\mathrm{Na}$ ture Neuroscience, 2, 1131-1136.

Serences, J. T., Schwarzbach, J., Courtney, S. M., Golay, X., \& YANTIS, S. (2004). Control of object-based attention in human cortex. Cerebral Cortex, 14, 1346-1357.

Smyth, M. M., \& Pendleton, L. R. (1989). Working memory for movements. Quarterly Journal of Experimental Psychology, 41A, 235-250.

Tresch, M. C., Sinnamon, H. M., \& Seamon, J. G. (1993). Double dissociation of spatial and object visual memory: Evidence from se- 
lective interference in intact human subject. Neuropsychologia, 31, 211-219.

Ungerleider, L. G., Galkin, T. W., \& Mishkin, M. (1983). Visuotopic organization of projections from striate cortex to inferior and lateral pulvinar in rhesus monkey. Journal of Comparative Neurology, 217, 137-157.

Ungerleider, L. G., \& Mishin, M. (1982). Two cortical visual systems. In D. J. Ingle, M. A. Goodale, \& R. J. Mansfield (Eds.), Analysis of visual behavior (pp. 549-586). Cambridge, MA: MIT Press.

VeCERA, S. P., \& FARAH, M. J. (1994). Does visual attention select objects or locations? Journal of Experimental Psychology: General, 123, 146-160.

\section{NOTES}

1. Note that when we used different-shaped objects during the shape tasks, it was impossible to place the Lego tile in the same, object-based position across all stimuli. Therefore, we randomly positioned the Lego tile during this task, ensuring that location information was noninforma- tive for solving the shape task, in a similar way that shape was noninformative for the location task.

2. Because we used the same response criterion across Experiments $2 \mathrm{~A}$ and $2 \mathrm{~B}$ for consistency, data from a large number of participants were excluded here. However, when we analyzed the data across all participants, we found the same effects as reported above with 12 participants - that is, no effect of primary or interference task but a significant interaction between these factors $[F(1,23)=21.68, p=.0001]$. This finding confirms a robust effect of task interference within the haptic domain.

3. We analyzed the data across all 24 participants and again found a significant interaction between the primary task and interference task factors $[F(1,23)=4.65, p=.05]$. This finding confirms a robust effect of task interference across modalities. We also found a main effect of modality $[F(1,23)=16.11, p<.01]$. Here, we found no evidence for a main effect of primary task $[F(1,23)<1]$. All other effects were nonsignificant.

(Manuscript received September 29, 2006; revision accepted for publication June 14, 2007.) 